\title{
Strategies to perform magnetic resonance imaging in infants and young children without sedation
}

\author{
Samantha G. Harrington ${ }^{1}$ (D) Camilo Jaimes $^{2} \cdot$ Kathryn M. Weagle ${ }^{1} \cdot$ Mary-Louise C. Greer $^{3,4} \cdot$ Michael S. Gee $^{1}$ \\ Received: 5 November 2020 / Revised: 25 January 2021 / Accepted: 16 March 2021 / Published online: 8 April 2021 \\ (C) The Author(s), under exclusive licence to Springer-Verlag GmbH Germany, part of Springer Nature 2021
}

\begin{abstract}
Given the increasing use of MRI in the pediatric population, the need for sedation in MRI performed in young children is a topic of growing importance. Although sedation is generally tolerated well by children, the financial and operational impacts of anesthesia on MRI workflow, as well as potential adverse effects of anesthetic medications, highlight the need to perform MRI in children without sedation whenever possible. This review focuses on current techniques to facilitate non-sedation MRI in children, including exam preparation with MRI simulation; asleep but not sedated techniques; awake and relaxed techniques using certified child life specialists, animal-assisted therapy, a child-friendly environment and in-scan entertainment; and non-sedated MRI protocol modifications such as shorter scan time, prioritizing sequences, reducing motion artifact, noise reduction, limiting use of gadolinium, employing an open MRI and modifying protocols.
\end{abstract}

Keywords Anesthesia $\cdot$ Child life specialist $\cdot$ Children $\cdot$ Magnetic resonance imaging $\cdot$ Sedation

\section{Introduction}

With the expanding applications and increasing utilization of pediatric MRI, the issue of sedation during pediatric MRI scans is growing in salience $[1,2]$. Challenges associated with sedation during MRI scans include prolonged waiting times for appointments, longer scan times and increased financial cost [3, 4]. More recently, coronavirus disease 2019 (COVID19) has complicated the use of general anesthesia, which now necessitates the use of personal protective equipment (PPE), comprehensive testing, and extensive decontamination, with most anesthesia MRI exams involving airway protection considered an aerosol-generating procedure [5, 6]. Additionally,

Samantha G. Harrington

sgharrington@partners.org

1 Department of Radiology, Massachusetts General Hospital, 55 Fruit St., Boston, MA 02114, USA

2 Boston Children's Hospital, Boston, MA, USA

3 Department of Diagnostic Imaging, Hospital for Sick Children, Toronto, ON, Canada

4 Department of Medical Imaging, University of Toronto, Toronto, ON, Canada use of anesthetic medications in children carries potential risks, including a small but non-zero risk of cardiorespiratory complications and adverse reactions [7]. Recent attention has focused on potential long-term neurologic effects of anesthetic medication exposure in infants, particularly those younger than 2 years who receive repeated anesthetic exposures during medical procedures $[8,9]$. In 2016, the United States Food and Drug Administration (FDA) placed black box warnings on certain anesthetic medications for children younger than 3 years and anesthetic exposure longer than $3 \mathrm{~h}$ because of these concerns [10]. One major confounder in quantifying anesthetic risk is the fact that almost all medical procedures performed in infants and young children, including MRI exams, are performed for medically necessary reasons and it is difficult to separate effects of anesthesia from those of the children's underlying medical conditions. Two recent prospective clinical trials following the neurologic development of infants and young children after a single anesthetic exposure for inguinal hernia repair found no significant impact on future neurologic development compared with control patients $[11,12]$. Nonetheless, there is a direct relationship between the length of sedated MRI exams and the dose of anesthetic medication administered to children, highlighting the need to minimize the use of anesthesia for MRI when possible and shorten MR exam duration [13]. 


\section{Preparation for the scan}

The first step is identifying children who might need sedation versus those who do not. Generally, children younger than 6 months can use the asleep but not sedated techniques reviewed later. From the age of 6 years, children might be able to follow directions and tolerate exams without the need for sedation. The most challenging period is 6 months to 6 years, which is why anesthesia is often required for children in this age range [14]. However, the unique circumstances of some children might necessitate the use of anesthesia outside this age range. For example, children older than 6 years with developmental delay, cerebral palsy or other neuromuscular condition, or status post a recent neurosurgical procedure, might not be able to adhere to breath-holding directions or lie still in the MR scanner without sedation.

The appropriate imaging protocol should be selected beforehand to plan for the length of the exam. Once a protocol is selected, there should be careful planning and coordination with the clinical providers and the family. If there is any concern that a child might not be able to tolerate a non-sedated exam, accommodations should be made for a subsequent sedated exam if needed (e.g., scheduling a sedation MRI timeslot after the non-sedation timeslot if available). There should be a system in place for a certified child life specialist, nurses, technologists and anesthesiologist to work together in coordination to prepare for the exam.

Preparing the child and family/caregivers for the exam is critical. Exam preparation, length and possible need for sedation should be reviewed with the caretakers, and the child should be prepared for the exam with age-appropriate techniques. One useful strategy is MRI simulation with a mock MRI experience $[4,15$, 16], which can be used to help familiarize children with the environment and anticipate whether they will be able to tolerate the exam. Video goggles or a cardboard viewer box can also be used (Fig. 1) to simulate the MRI experience. Other tools include coloring books or storybooks, visiting the scanner/waiting room and watching videos beforehand [17].

\section{Asleep but not sedated}

For children younger than 6 months, the asleep but not sedated techniques are often successful. Non-nutritive sucking and sucrose have been shown to reduce infant discomfort, with one center reporting success in $89 \%$ of cases [18]. Coupling feeding and swaddling can help induce sleep during the exam (Fig. 2) [19]. Swaddling can be helpful in reducing motion degradation during the exam. Extensive sleep deprivation is not recommended prior to the exam because of increased burden

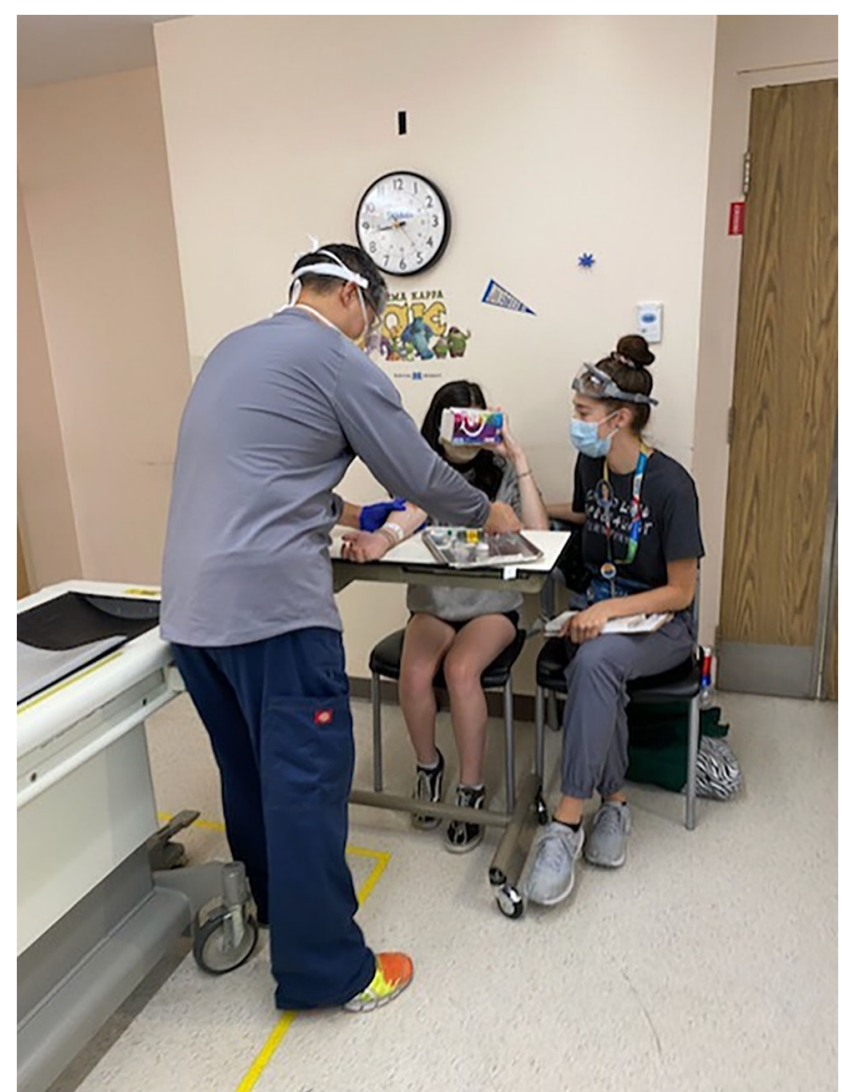

Fig. 1 Photo of a child with a cardboard viewer box that can be used to simulate the MRI experience and prepare older children for their MRI scan. If virtual-reality goggles are not available, a cardboard box in combination with a smartphone can be used to simulate the experience. Please note that this photo was taken during the coronavirus disease 2019 (COVID-19) pandemic, when masks were regularly worn. Credit: CHISIL.ca and the SickKids virtual reality program

on caretakers, as well as increased nursing care requirements following the procedure [20]. However, it is recommended to keep the child awake $3-4 \mathrm{~h}$ prior to the exam, with one feed withheld [21] to facilitate sleeping after feeding. To encourage sleep, lights should be kept dim and attempts at noise reduction should be made. Evening timeslots might also be beneficial for families and help decrease crowding and noise in the area around the MR scanner [22].

\section{Awake and relaxed}

\section{Certified child life specialist}

For children 6 years and older, the awake and relaxed techniques can lead to a successful non-sedated scan (Fig. 3). An essential component to the exam is the certified child life specialist. A child life specialist helps "infants, children, youth and families cope with the stress and uncertainty of acute and 

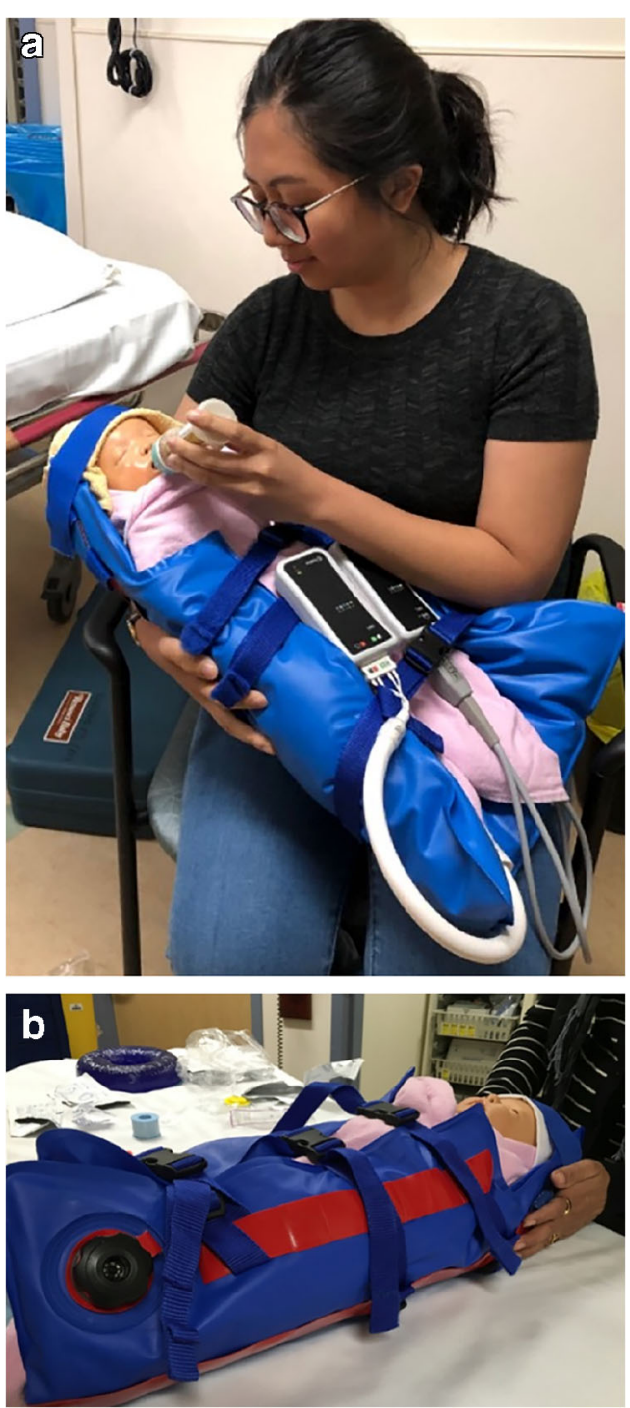

Fig. 2 Feed-and-sleep nonsedation MRI. a, b Clinical images. Feeding the baby right before the scan (a) in combination with swaddling (b) to induce sleep can allow neonatal MRI to be performed without sedation chronic illness, injury, trauma, disability, loss and bereavement" [23]. The child life specialist plays a critical role in preparing the child before the exam and supporting the child during the exam using age-appropriate techniques [24]. A hospital radiology department having child life specialist has been shown to reduce the need for anesthesia [25]. For awake and relaxed MRI, child life specialists often meet with children and caregivers ahead of time to review MRI workflow and answer questions, then on the day of the procedure they go through MRI simulation with the child prior to the MRI scan. Child life specialists are also available throughout the scan to help reassure the child and caregiver and provide support.

\section{Animal-assisted therapy}

A newer technique being incorporated into radiology departments is animal-assisted therapy (Fig. 4). A study by Perez et al. [26] showed significant improvement in patient anxiety without detrimental impact on the duration of MRI scan time or image quality. Therapy dogs can be used to calm children in the waiting room, during intravenous line placement and when escorting the child to the MRI. Therapy dog handlers work with child life specialists and the radiology team to ensure the dog remains leashed and calm during the interaction. Therapy dogs are screened for implants and trained to work in the hospital setting with an experienced handler.

\section{Child-friendly environment}

Creating a child-friendly environment makes for a welcoming experience for children (Fig. 5). Using colorful and playful imagery in the waiting room and MRI suite can also be useful, especially for school-age children. A
Fig. 3 Certified child life specialists are integral to setting up a successful non-sedated MRI exam. Among their roles are providing support and education to children and their families and explaining what to expect with exams (often using portable electronic devices, as shown)

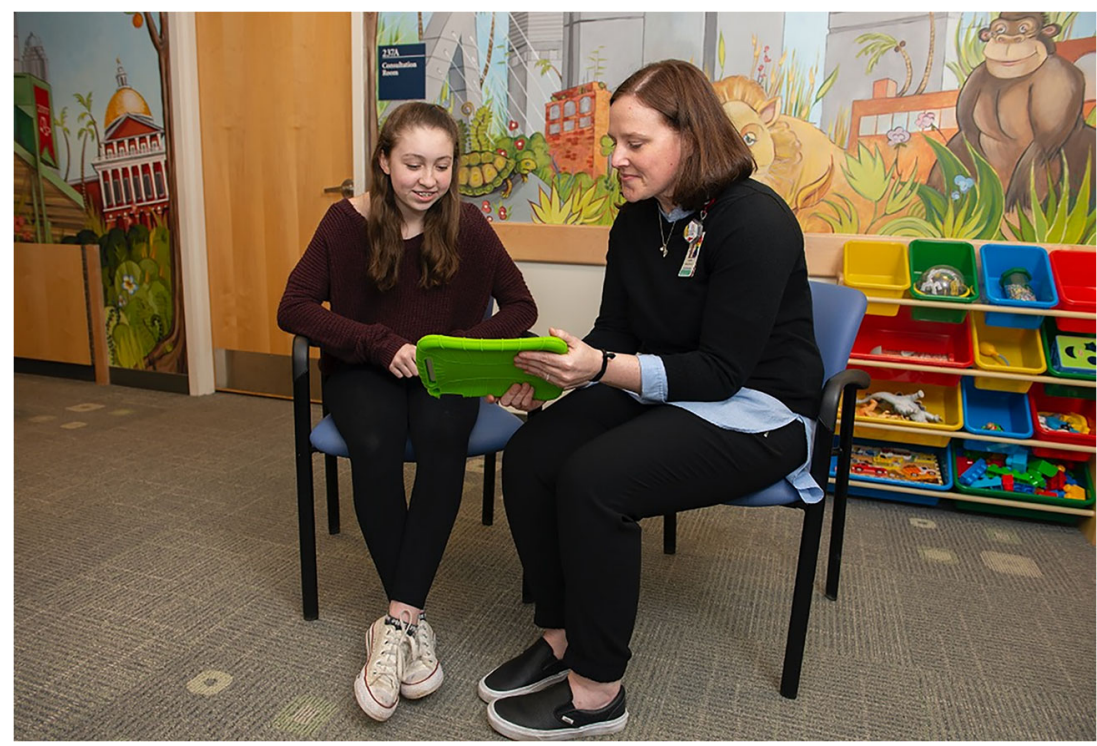




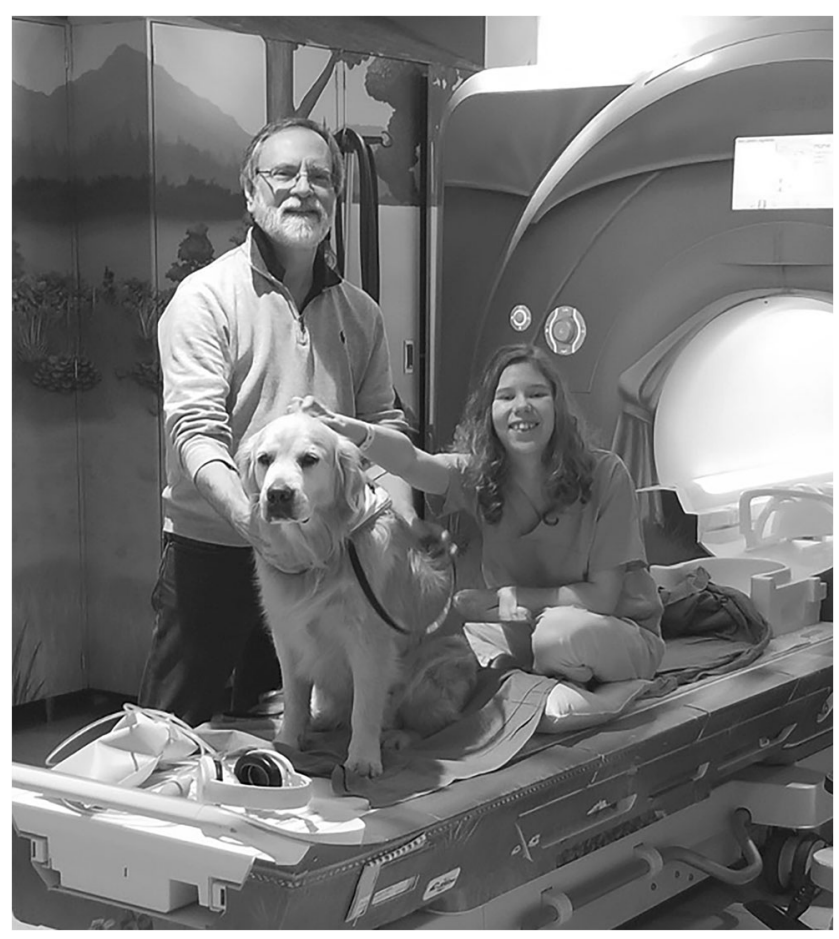

Fig. 4 Animal-assisted therapy is useful for relaxing older children. Therapy dogs can accompany children into the MR scanner and make them feel relaxed and comfortable before the start of the scan

child-friendly environment helps to make the scanner appear less foreign and mechanical, and more inviting and familiar to children. If possible, MRI scanners can be designated as pediatric scanners so that permanent decorations can be applied to the suite. However, in cases where an MRI scanner is used for both adults and pediatric patients, scanner modifications might need to be digital (e.g., projected scenes with music) so that they can be changed depending on patient age and interests.

\section{In-scan entertainment}

During the MRI scan, children can be provided with an MRI-compatible audio-visual system for entertainment (Fig. 6). The use of a video system has been shown to reduce need for sedation by $18 \%$, as well as lead to decreased scan time [27]. Overall, use of these systems is well-received by children and is associated with successful awake MRI exams [22, 28]. Video goggles fit snugly against the child's skin and can make some children feel warm during a long MRI exam. Additionally, goggles might be incompatible with certain head or neck coils. In these cases, video glasses with prisms that reflect video images from an external monitor might be preferable. Also, watching videos can make some children more stimulated and less willing to lie still. In these cases, having children listen to music using headphones without a
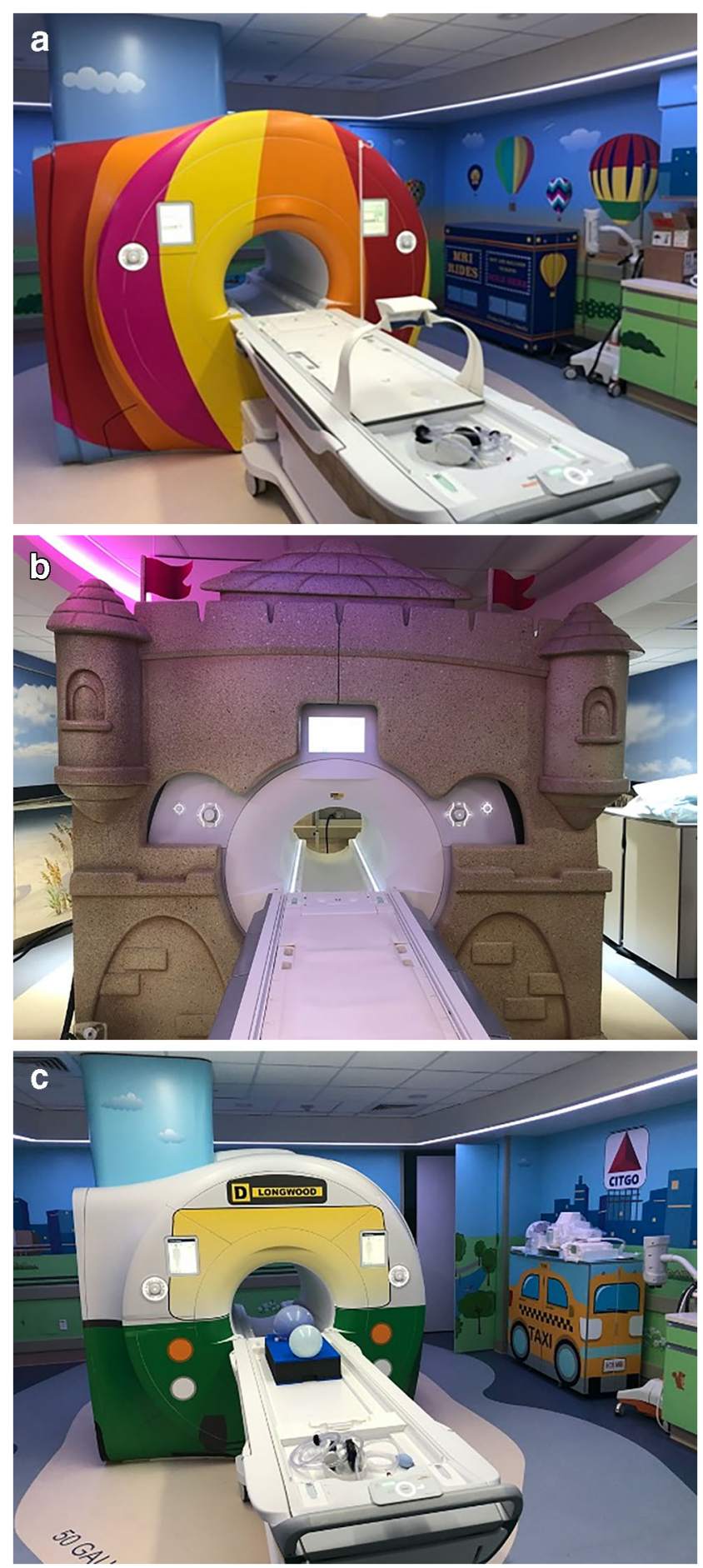

Fig. 5 A child-friendly environment can be welcoming to patients. a-c Examples of child-friendly MRI scanners include a bright hot balloon theme (a), sandcastle theme (b) and Boston city landscape with the MRI as a trolley (c)

video feed might be preferable. An integrated workflow for non-sedation pediatric MRI including patient screening, preparation for the MRI and management is shown in Fig. 7. 
Fig. 6 MRI-compatible audiovisual equipment can be used as in-scan entertainment. Clinical image shows a child using goggles that supply a video feed from DVD (digital versatile disc) movies while audio input goes through headphones

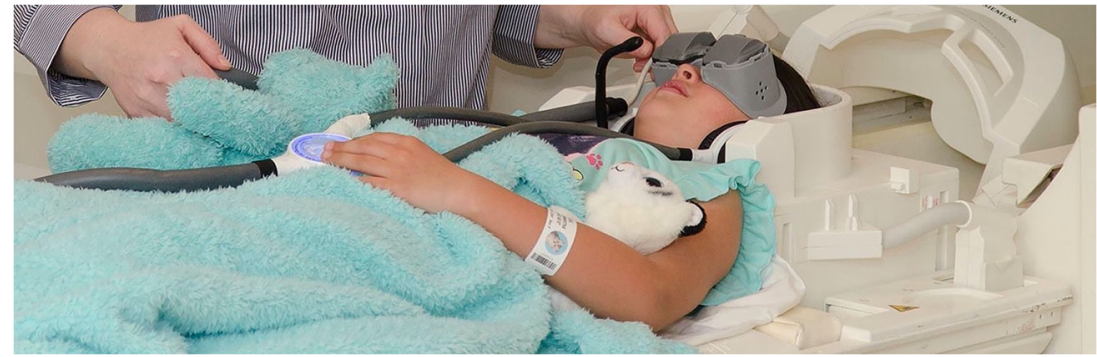

\section{Magnetic resonance imaging protocol modifications}

\section{Shorter scanning time}

Reducing the time the child needs to be in the scanner increases the likelihood that the child will able to tolerate the exam without non-diagnostic images, aborted exam or requirement for anesthesia [22]. Additionally, the longer the scan time, the greater the medication dose children receive during sedated exams [13]. Examples of fast MRI techniques include parallel imaging, simultaneous excitation MR techniques (exciting multiple slices simultaneously using a composite radiofrequency pulse), compressed-sensing MRI reconstruction and automated protocol selection techniques [29-31].

Another strategy to decrease scan time is to leverage optimal MRI hardware, such as a 3-tesla (T) scanner with high count phased-array coils, to decrease the number of excitations while maintaining a reasonable signal-to-noise ratio (SNR). A prospective study on the diagnostic equivalency of single-excitation turbo spin-echo T2-weighted and fluidattenuated inversion recovery (FLAIR) sequences in pediatric neuroimaging showed non-inferiority of the optimized sequences, while providing 50\% decrease in scan time [32]. This approach offers the advantage of being vendor-neutral and independent of scanner operating system, factors that can often limit the implementation and dissemination of novel sequences.

\section{Prioritization of sequences}

Sequences should be prioritized in order of clinical importance. It is also preferable to run louder sequences such as diffusion-weighted imaging using echoplanar imaging at the end to minimize waking an infant if using the feed-andswaddling non-sedate technique. Knowing the clinical question and situation prior to the exam is essential. For example, an MRI for query of the abdomen for appendicitis can typically be performed in 1-2 min using single-shot fast spin-echo T2-weighted images in multiple planes (vendor-specific names include half-Fourier acquisition single-shot turbo spin echo [HASTE], single-shot fast spin echo [SSFSE] or singleshot turbo spin echo [SSTSE]). Additional pulse sequences can be acquired afterward with superior soft-tissue contrast for evaluating the appendix tip and assess for perforation, if the single-shot T2-weighted images are equivocal and the
Fig. 7 Chart shows potential workflow for optimizing a nonsedated MRI scan for children: screening, preparation and management. $C C L S$ certified child life specialist

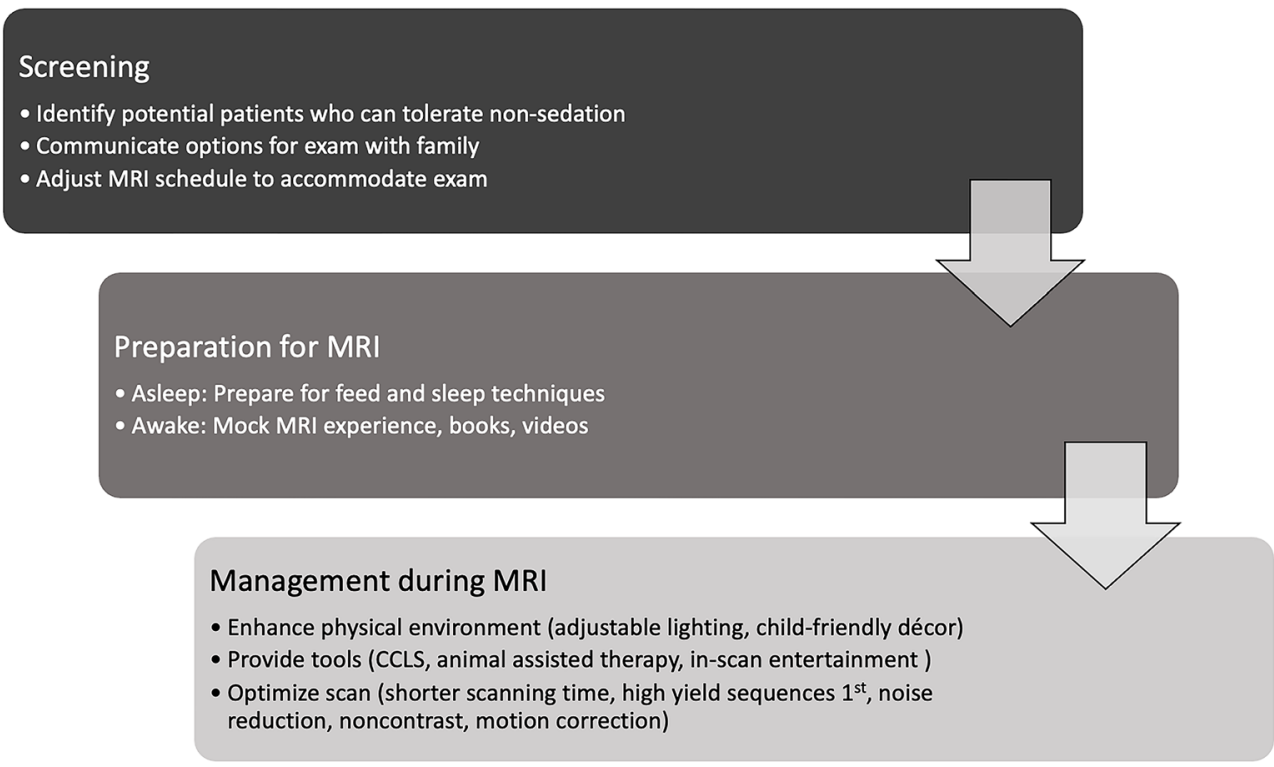


child is able to tolerate it. Thus, monitoring of the exam is crucial to ensure images are adequate.

\section{Motion artifact reduction}

A variety of strategies can be used to reduce motion, which in turn increases the likelihood of a successful scan in a shorter time. Using non-breath-hold sequences relying on steady, quiet respiration reduces the need for the child to adhere to strict directions. Techniques to correct motion artifact include swapping the phase and frequency coding directions, tissue suppression with use of saturation bands, respiratory triggering and signal averaging [7]. Radial k-space sampling techniques can also help decrease respiratory motion artifacts by dispersing phase-encoding respiratory artifacts along multiple incoherent planes, while preserving image contrast through oversampling of the center of k-space [29]. Variations of radial techniques include two-dimensional (2-D) radial T2weighted sequences (PROPELLER [periodically rotated overlapping parallel lines with enhanced reconstruction], BLADE, MultiVANE); stack of stars radial gradient recalled echo T1weighted sequences; and full three-dimensional (3-D) radial sequences (StarVIBE [volumetric interpolated breath-hold examination], 3-D VANE, LAVA [liver acquisition with volume acquisition] star). These sequences have been shown to reduce motion artifacts in pediatric MRI exams, in both awake and sedated patients (Fig. 8) [30].

\section{Acoustic noise reduction}

Magnetic resonance imaging scans can produce sound pressures as high as $120 \mathrm{~dB}$. Reducing noise is helpful for both sleep and awake techniques. Dean et al. [33] showed a successful sound reduction from $120 \mathrm{~dB}$ to $85 \mathrm{~dB}$ using ear plugs, insulating foam and reduced slew rates for T1-weighted spoiled gradient echo (SPGR) and inversion-prepared SPGR, balanced steady-state free precession sequences. Using these techniques added approximately $30 \mathrm{~min}$ of scan preparation time [33].

\section{Limited use of gadolinium}

The use of gadolinium requires establishing intravenous access, which can be uncomfortable for children. Furthermore, there are a variety of safety concerns regarding gadolinium including allergic reactions. Recent studies have raised the concern for gadolinium deposition in tissues; however, the clinical significance of this finding is uncertain [34]. It is feasible that certain pediatric MRI protocols can be obtained without the use of gadolinium, such as MR enterography, musculoskeletal infection or acute appendicitis protocols [35-39].
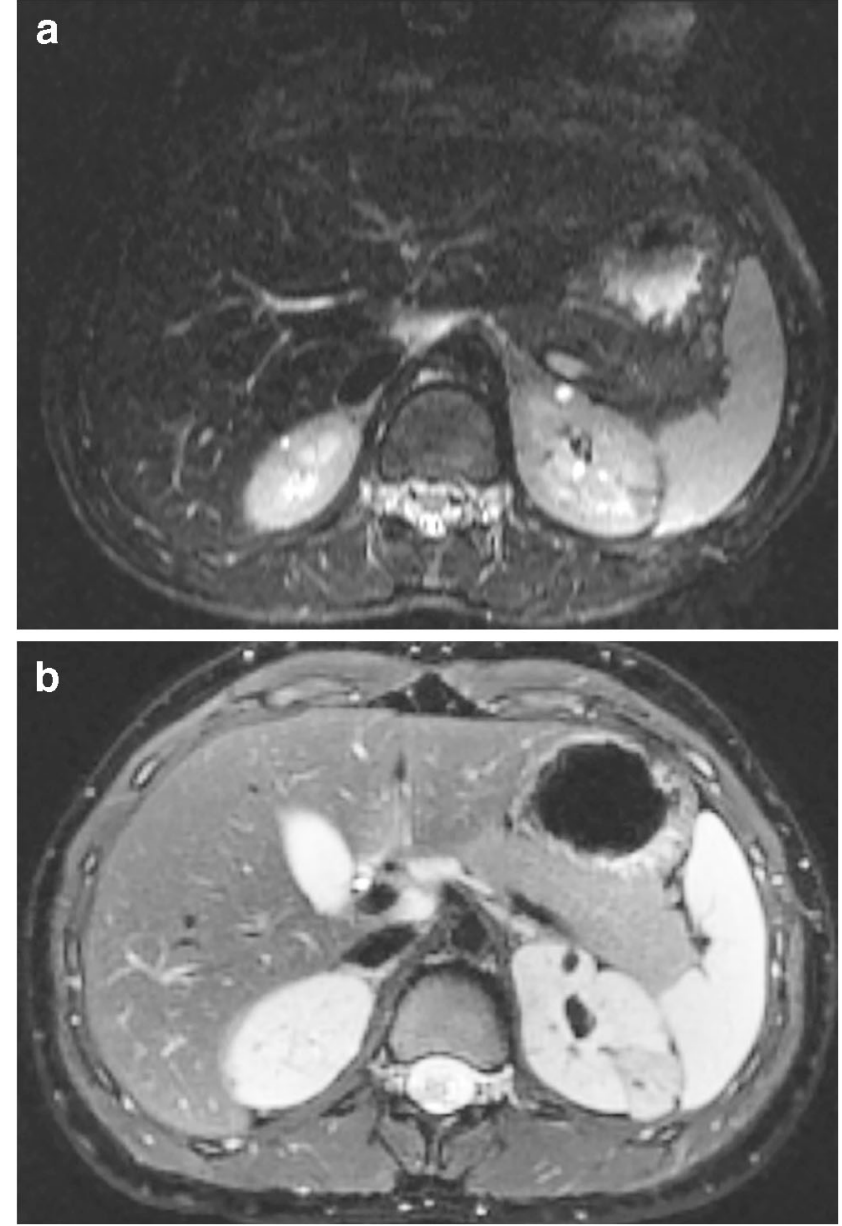

Fig. 8 Fast imaging techniques in a 10 -year-old boy with tuberous sclerosis. a, b Axial T2-W fast spin-echo Cartesian fat-saturated MR image (a), and follow-up surveillance MRI 1 year later with axial BLADE sequence (b). The BLADE sequence shows much reduced respiratory motion. The BLADE sequence helps reduce motion artifact by using radial $\mathrm{k}$-space technique

\section{Open magnetic resonance imaging}

An open MRI can reduce claustrophobia and might be better tolerated by older children who are awake and relaxed [40]. However, open MRI scanners acquire images at lower field strength compared with standard 1.5-T and 3.0-T clinical scanners, leading to increased scan times and reduced spatial/contrast resolution. Careful consideration should be given to the diagnostic image quality associated with an open MRI exam and, if not adequate to answer the clinical question, a standard field strength MRI with anesthesia should be considered instead.

\section{Proposed neuro and body short protocols}

Protocols can be tailored to children's needs by focusing on shorter scanning time, motion reduction, noise reduction and non-contrast exam. An example of a non-contrast appendicitis protocol includes axial and coronal T2-weighted single-shot 
fast spin-echo (FSE) sequences with and without fat saturation [35]. An example non-contrast abbreviated brain protocol includes axial and coronal T2-weighted single-shot FSE protocols with optional diffusion-weighted imaging (axial and coronal) and sagittal FSE [41].

\section{Future developments}

Continued advances in MR acceleration techniques, prospective motion correction and image reconstruction are expected to continue to shorten MR protocols that produce diagnosticquality images and make MRI easier to tolerate for nonsedated children. In addition, harmonization of abbreviated MR protocols that allow the same protocols to be used in sedated and non-sedated children are expected to facilitate ease of scheduling both types of exams and the ability to switch from one category to the other based on how children fare during MR simulation. Additionally, sparse sampling combined with deep-learning reconstruction methods might be a future potential option.

\section{Conclusion}

Creating a successful non-sedated exam for pediatric patients requires advanced planning. Pediatric MR services should have a non-sedated workflow that includes patient screening, preparation for the MRI and management during the scan. Identifying potential non-sedated patients and preparing the child and family is critical. The feed-and-sleep technique can be successful for children younger than 6 months. The awake and relaxed technique can be used with children older than 6 years. Strategies to promote a successful awake scan include close coordination with child life specialists, animal assisted-therapy, a child-friendly environment and entertainment during the exam. Modifying existing protocols to shorten scan time, prioritize certain sequences, correct for motion artifact, promote noise reduction, limit use of gadolinium and use open MRIs might all contribute to a successful non-sedated exam.

\section{Declarations}

Conflicts of interest Dr. Greer has a research grant from AbbVie as well as speaker's honoraria from AbbVie/Samsung.

\section{References}

1. Scheinfeld MH, Moon J-Y, Fagan MJ et al (2017) MRI usage in a pediatric emergency department: an analysis of usage and usage trends over 5 years. Pediatr Radiol 47:327-332
2. Uffman JC, Tumin D, Raman V et al (2017) MRI utilization and the associated use of sedation and anesthesia in a pediatric ACO. J Am Coll Radiol 14:924-930

3. Malviya S, Voepel-Lewis T, Eldevik OP et al (2000) Sedation and general anaesthesia in children undergoing MRI and CT: adverse events and outcomes. Br J Anaesth 84:743-748

4. Vanderby SA, Babyn PS, Carter MW et al (2010) Effect of anesthesia and sedation on pediatric MR imaging patient flow. Radiology 256:229-237

5. Zucco L, Levy N, Ketchandji D et al (2020) An update on the perioperative considerations for COVID-19 severe acute respiratory syndrome coronavirus-2 (SARS-CoV-2). APSF Newsletter. https://www.apsf.org/news-updates/perioperative-considerationsfor-the-2019-novel-coronavirus-covid-19/. Accessed 27 Sep 2020

6. Ertl-Wagner BB, Lee W, Manson DE et al (2020) Preparedness for the COVID-19 pandemic in a tertiary pediatric radiology department. Pediatr Radiol 50:1059-1068

7. Jaimes C, Gee MS (2016) Strategies to minimize sedation in pediatric body magnetic resonance imaging. Pediatr Radiol 46:916-927

8. Flick RP, Katusic SK, Colligan RC et al (2011) Cognitive and behavioral outcomes after early exposure to anesthesia and surgery. Pediatrics 128:e1053-e1061

9. Wang X, Xu Z, Miao C-H (2014) Current clinical evidence on the effect of general anesthesia on neurodevelopment in children: an updated systematic review with meta-regression. PLoS One 9: e85760

10. Derderian CA, Szmuk P, Derderian CK (2017) Behind the black box: the evidence for the U.S. Food and Drug Administration warning about the risk of general anesthesia in children younger than 3 years. Plast Reconstruct Surg 140:787-792

11. Sun LS, Li G, Miller TLK et al (2016) Association between a single general anesthesia exposure before age 36 months and neurocognitive outcomes in later childhood. JAMA 315:23122320

12. McCann ME, de Graaff JC, Dorris L et al (2019) Neurodevelopmental outcome at 5 years of age after general anaesthesia or awake-regional anaesthesia in infancy (GAS): an international, multicentre, randomised, controlled equivalence trial. Lancet 393:664-677

13. Machado-Rivas F, Leitman E, Jaimes C et al (2020) Predictors of anesthetic exposure in pediatric MRI. AJR Am J Roentgenol. https://doi.org/10.2214/AJR.20.23601

14. Edwards AD, Arthurs OJ (2011) Paediatric MRI under sedation: is it necessary? What is the evidence for the alternatives? Pediatr Radiol 41:1353

15. de Bie HMA, Boersma M, Wattjes MP et al (2010) Preparing children with a mock scanner training protocol results in high quality structural and functional MRI scans. Eur J Pediatr 169:1079-1085

16. Carter AJ, Greer M-LC, Gray SE, Ware RS (2010) Mock MRI: reducing the need for anaesthesia in children. Pediatr Radiol 40: 1368-1374

17. Törnqvist E, Månsson $\AA$, Hallström I (2014) Children having magnetic resonance imaging: a preparatory storybook and audio/visual media are preferable to anesthesia or deep sedation. J Child Health Care 19:359-369

18. Carbajal R, Chauvet X, Couderc S, Olivier-Martin M (1999) Randomised trial of analgesic effects of sucrose, glucose, and pacifiers in term neonates. BMJ 319:1393-1397

19. Hansen SS (2009) Feed-and-sleep: a non-invasive and safe alternative to general anaesthesia when imaging very young children. Radiographer 56:5-8

20. Shields CH, Johnson S, Knoll J et al (2004) Sleep deprivation for pediatric sedated procedures: not worth the effort. Pediatrics 113: 1204-1208

21. Windram J, Grosse-Wortmann L, Shariat M et al (2012) Cardiovascular MRI without sedation or general anesthesia using 
a feed-and-sleep technique in neonates and infants. Pediatr Radiol 42:183-187

22. Jaimes C, Robson CD, Machado-Rivas F et al (2020) Success of non-sedated neuroradiological MRI in children 1 to 7 years old. AJR Am J Roentgenol. https://doi.org/10.2214/AJR.20.23654

23. Association of Child Life Professionals (2021) The child life profession. What is a certified child life specialist? Association of Child Life Professionals website. https://www.childlife.org/the-child-lifeprofession. Accessed 5 Sep 2020

24. McGee K (2003) The role of a child life specialist in a pediatric radiology department. Pediatr Radiol 33:467-474

25. Durand DJ, Young M, Nagy P et al (2015) Mandatory child life consultation and its impact on pediatric MRI workflow in an academic medical center. J Am Coll Radiol 12:594-598

26. Perez M, Cuscaden C, Somers JF et al (2019) Easing anxiety in preparation for pediatric magnetic resonance imaging: a pilot study using animal-assisted therapy. Pediatr Radiol 49:1000-1009

27. Harned RK II, Strain JD (2001) MRI-compatible audio/visual system: impact on pediatric sedation. Pediatr Radiol 31:247-250

28. Lemaire C, Moran GR, Swan H (2009) Impact of audio/visual systems on pediatric sedation in magnetic resonance imaging. $\mathrm{J}$ Magn Reson Imaging 30:649-655

29. Kozak BM, Jaimes C, Kirsch J, Gee MS (2020) MRI techniques to decrease imaging times in children. Radiographics 40:485-502

30. Balza R, Jaimes C, Risacher S et al (2019) Impact of a fast freebreathing 3-T abdominal MRI protocol on improving scan time and image quality for pediatric patients with tuberous sclerosis complex. Pediatr Radiol 49:1788-1797

31. Tabari A, Machado-Rivas F, Kirsch JE et al (2020) Performance of simultaneous multi-slice accelerated diffusion-weighted imaging for assessing focal renal lesions in pediatric patients with tuberous sclerosis complex. Pediatr Radiol 51:77-85

32. Jaimes C, Yang E, Connaughton P et al (2020) Diagnostic equivalency of fast T2 and FLAIR sequences for pediatric brain MRI: a pilot study. Pediatr Radiol 50:550-559
33. Dean DC, Dirks H, O'Muircheartaigh J et al (2014) Pediatric neuroimaging using magnetic resonance imaging during non-sedated sleep. Pediatr Radiol 44:64-72

34. Gale EM, Caravan P, Rao AG et al (2017) Gadolinium-based contrast agents in pediatric magnetic resonance imaging. Pediatr Radiol 47:507-521

35. Anderson MA, Harrington SG, Kozak BM, Gee MS (2020) Strategies to reduce the use of gadolinium-based contrast agents for abdominal MRI in children. AJR Am J Roentgenol 214:1054 1064

36. Alsharief AN, Martinez-Rios C, Hopyan S et al (2019) Usefulness of diffusion-weighted MRI in the initial assessment of osseous sarcomas in children and adolescents. Pediatr Radiol 49:1201-1208

37. Markhardt BK, Woo K, Nguyen JC (2019) Evaluation of suspected musculoskeletal infection in children over 2 years of age using only fluid-sensitive sequences at MRI. Eur Radiol 29:5682-5690

38. Trofimova A, Kadom N (2019) Added value from abbreviated brain MRI in children with headache. AJR Am J Roentgenol 212: 1348-1353

39. Covelli JD, Madireddi SP, May LA et al (2018) MRI for pediatric appendicitis in an adult-focused general hospital: a clinical effectiveness study - challenges and lessons learned. AJR Am J Roentgenol 212:180-187

40. Munn Z, Moola S, Lisy K et al (2015) Claustrophobia in magnetic resonance imaging: a systematic review and meta-analysis. Radiography 21:e59-e63

41. Barkovich MJ, Xu D, Desikan RS et al (2018) Pediatric neuro MRI: tricks to minimize sedation. Pediatr Radiol 48:50-55

Publisher's note Springer Nature remains neutral with regard to jurisdictional claims in published maps and institutional affiliations. 